\title{
A Two-Dimensional Theory of Fracture Propagation
}

M.A. Biot, Consultant

L. Massé, Mobil Research \& Development Co.

W.L. Medlin, Mobil Research \& Development Co.

\begin{abstract}
Summary. A basic theory of two-dimensional (2D) fracture propagation has been developed with a Lagrangian formulation combined with a virtual work analysis. Fluid leakoff is included by the assumption that an incompressible filtrate produces a piston-like displacement of a compressible reservoir fluid with a moving boundary between the two. Poiseuille flow is assumed in the fracture. We consider both Newtonian and nonNewtonian fluids with and without wall building. For non-Newtonian fluids, we assume the usual power-law relation between shear stress and shear rate. The Lagrangian formulation yields a pair of nonlinear equations in $L_{f}$ and $b_{f}$, the fracture length and half-width. By introducing a virtual work analysis, we obtain a single equation that can be solved numerically. For non-wall-building fluids, it predicts much higher leakoff rates than existing methods. The Lagrangian method also allows nonelastic phenomena, such as plasticity, to be included. A practical computer program developed from this theory has been used for more than 10 years to design fracturing treatments in oil and gas reservoirs in Canada, California, the midcontinent and Rocky Mountain areas, the U.S. gulf coast, the North Sea, and in northern Germany. In most of these applications, it has predicted fracture dimensions that have been in line with production experience. Optimization methods based on this program led to very large fracturing treatments in low-permeability gas sands that were forerunners of massive fracturing treatments in tight gas sands. Specific examples in which this method was used to design fracturing programs in large gas fields in Kansas and Texas are discussed.
\end{abstract}

\section{Introduction}

We present here a new approach to the $2 \mathrm{D}$ problem of fracture propagation based on Lagrangian methods. The Lagrangian formulation has been applied to a variety of problems in physics and chemistry. ${ }^{1-3}$ To the best of our knowledge, however, this is the first application to fracture mechanics.

The Lagrangian formulation is based on the classical form of Lagrange's equations. As applied here, it produces a basic equation that expresses the balance between work expended and work done in propagating a 2D crack.

Existing theories of crack propagation have all been developed by the application of equations from classical elasticity theory. This approach assumes linear elastic behavior of the reservoir rock and ignores surface energy considerations at the crack tip and plastic deformation effects. Leakoff, if it is included, is treated as an independent process and merged with the crack propagation problem by iterative methods that assume self-consistency. Some well-known examples of this approach have been presented by Zheltov and Khristianovich, ${ }^{4,5}$ Perkins and Kern, ${ }^{6}$ Nordgren, ${ }^{7}$ Geertsma and de Klerk, ${ }^{8}$ Daneshy, ${ }^{9}$ Le Tirant and Dupuis, ${ }^{10}$ and Cleary. ${ }^{11,12}$ Geertsma and Haafkens ${ }^{13}$ have compared many of the results of these theories.

A more general approach, the Lagrangian method is not restricted to elastic behavior, and leakoff can be included as an integral part of the formulation. We include leakoff by assuming a piston-like displacement of com-

Copyright 1986 Society of Petroleum Engineers pressive reservoir fluid by an incompressible fracture fluid filtrate with a moving boundary between the two.

The Lagrangian formulation yields a pair of nonlinear differential equations in fracture length $L_{f}$ and fracture half-width $b_{f}$, which are reduced to a single equation in $L_{f}$ by introduction of a virtual work analysis. This equation can be solved numerically and can be used with other relations to obtain fracture dimensions and injection pressure as a function of time at constant injection rate. Experimental laboratory measurements reported previously ${ }^{14}$ confirm basic results obtained from such computations.

The Lagrangian formulation presented here has been used for many years in our field operations to predict fracture dimensions. It has provided a means to plan and to optimize fracture treatments in a variety of field operations. Some specific field applications will be discussed.

\section{The Lagrangian Formulation}

The usual form of Lagrange's equations from classical mechanics is ${ }^{15}$

$$
\frac{\mathrm{d}}{\mathrm{d} t}\left(\frac{\partial L}{\partial \dot{q}_{i}}\right)-\frac{\partial L}{\partial q_{i}}=\bar{Q}_{i},
$$

where $L=E_{k}-E_{p}$. Because fracture propagation occurs under near static conditions, the kinetic energy term, $E_{k}$, can be neglected in our application. $\bar{Q}_{i}$ includes all forces that are not derived from a potential function. It can be 


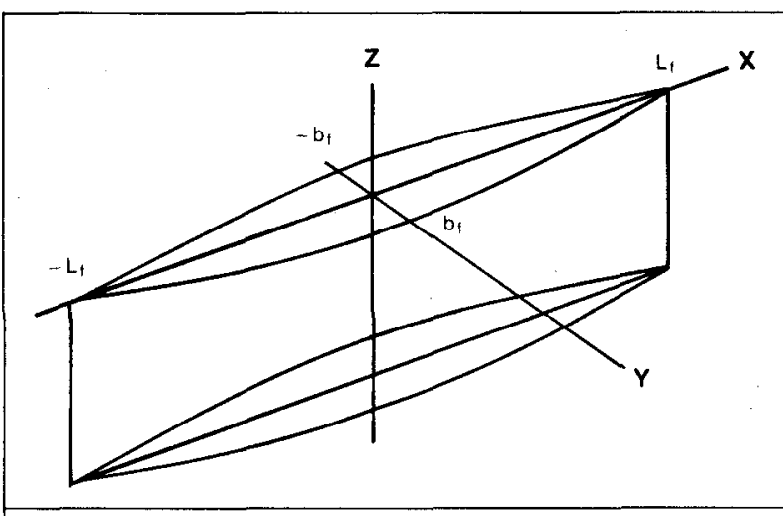

Fig. 1-Two-dimensional fracture of unit height.

divided into frictional forces derived from a dissipation function $D$ and all remaining forces $Q_{i}$. Thus

$$
\bar{Q}_{i}=-\frac{\partial D}{\partial \dot{q}_{i}}+Q_{i}
$$

Making these substitutions reduces Eq. 1 to

$$
\frac{\partial E_{p}}{\partial q_{i}}+\frac{\partial D}{\partial \dot{q}_{i}}=Q_{i},
$$

which is the form of Lagrangian equations we will use.

We will apply these equations to the problem of $2 \mathrm{D}$ fracture propagation. The assumed fracture geometry is shown in Fig. 1. The fracture has constant height along $x$ and constant width along $z$ over its height. Thus the problem is assumed to be one of plane strain in the $x-y$ plane. We choose two coordinates, $q_{1}=L_{f}$ and $q_{2}=b_{f}$. We assume that the fracture extends from $-L_{f}$ to $L_{f}$ and that its width is given by

$$
b=2 b_{f} \mathrm{f}\left(\frac{x}{L_{f}}\right)=2 b_{f} \mathrm{f}\left(L_{D}\right) .
$$

The function $\mathrm{f}\left(L_{D}\right)$ specifies the shape of the crack. It has the following properties: $\mathrm{f}\left(-L_{D}\right)=\mathrm{f}\left(L_{D}\right) ; \mathrm{f}(0)=1$; $f(1)=0$; and $f$ is monotone for $0 \leq L_{D}<1$. Because of this symmetry, we need to consider only the half-plane $x>0$ in Fig. 1.

In this case, the Lagrangian equations are

$$
\frac{\partial E_{p}}{\partial L_{f}}+\frac{\partial D}{\partial \dot{L}_{f}}=Q_{1}
$$

and

$$
\frac{\partial E_{p}}{\partial b_{f}}+\frac{\partial D}{\partial \dot{b}_{f}}=Q_{2}
$$

To apply these equations, we must find the potential function $E_{p}$, the dissipation function $D$, and the generalized forces $Q_{1}$ and $Q_{2}$ for the fracture in Fig. 1. These are derived in Appendix A for the simplest case of a Newtonian fracture fluid in an elliptical crack without leakoff.

In this simplest case, $E_{p}, D$, and $Q_{i}$ have easily recognized physical meanings. The potential energy $E_{p}$ is the work required to build up an internal pressure in the crack volume. $D$ is the rate of energy dissipation caused by frictional forces arising from fluid flow down the crack. The generalized forces $Q_{1}$ and $Q_{2}$ include all of the forees requircd to increase the crack volume and to generate new crack surface at the fracture tip.

Expressions for $E_{p}, D, Q_{1}$, and $Q_{2}$ are given by Eqs. A-5, A-15, and A-23. Substituting these results in Eqs. $5 \mathrm{a}$ and $5 \mathrm{~b}$ gives the basic differential equations

$$
\begin{aligned}
6 \mu(A+B) & \frac{L_{f}^{2} \dot{b}_{f}}{b_{f}^{2}}+\dot{6} \mu(A+2 B+C) \frac{L_{f} \dot{L}_{f}}{b_{f}} \\
& =2 \beta p_{e} b_{f}-E \ldots \ldots \ldots \ldots
\end{aligned}
$$

and

$$
\begin{aligned}
2 K \gamma b_{f} & +6 \mu A \frac{L_{f}{ }^{3} \dot{b}_{f}}{b_{f}{ }^{3}}+6 \mu(A+B) \frac{L_{f}{ }^{2} \dot{L}_{f}}{b_{f}^{2}} \\
& =2 \beta p_{e} L_{f} \ldots \ldots \ldots \ldots \ldots \ldots \ldots
\end{aligned}
$$

We seek solutions to these equations of the form

$$
\begin{aligned}
& L_{f}=C_{1} t^{h}, \\
& b_{f}=C_{2} t^{m}, \\
& p_{e}=C_{3} t^{r},
\end{aligned}
$$

and

$$
q_{t e}=C_{4} t^{s}
$$

Substitution of Eq. 8 into Eqs. 6 and 7 gives $h=2 / 3$, $m=1 / 3, r=-1 / 3$, and $s=0$, or

$$
\begin{aligned}
& L_{f}=C_{1} t^{2 / 3}, . \\
& b_{f}=C_{2} t^{1 / 3}, . \\
& p_{e}=C_{3} t^{-1 / 3},
\end{aligned}
$$

and

$$
q_{t e}=C_{4} .
$$

Thus the form of the solution we have chosen corresponds to the case of constant injection rate, which is the one of practical interest.

The constants $C_{1}, C_{2}$, and $C_{3}$ are evaluated in Appendix $\mathrm{B}$. The results obtained there allow us to express $L_{f}, b_{f}$, and $p_{e}$ as explicit functions of time for the case of no leakoff. We also show in Appendix B how these results can be used under appropriate assumptions to derive the width equation obtained by Geertsma and de Klerk. ${ }^{8}$ 
We see from Eqs. 9 that two combinations of variables are independent of time. These are $p_{e} L_{f} / b_{f}$ and $b_{f}{ }^{2} / L_{f}$. There are two extreme cases for which these combinations reduce to simple results, as shown in Appendix B. For large values of $\mu q_{t e}$,

$$
\frac{p_{e} L_{f}}{b_{f}}=\frac{C_{1} C_{3}}{C_{2}}=\frac{K \gamma}{\beta}\left(\frac{6 A+7 B+2 C}{3 A+2 B}\right) \ldots \ldots
$$

and

$$
\frac{b_{f}^{2}}{L_{f}}=\frac{C_{2}^{2}}{C_{1}}=\sqrt{\left(\frac{3 B+2 C}{\beta \gamma}\right) \frac{\mu q_{t e}}{K}} .
$$

For small values of $\mu q_{t e}$,

$$
\frac{p_{e} L_{f}}{b_{f}}=\frac{K \gamma}{\beta}+\frac{6 \mu q_{t e}}{\beta^{2}}\left(A+\frac{2}{3} B\right) \frac{K^{2} \gamma^{2}}{E^{2}}
$$

and

$$
\frac{b_{f}^{2}}{L_{f}}=\frac{E}{2 K \gamma}
$$

Shape of the Crack. The preceding analysis has assumed that the crack shape is given. However, this is not a prerequisite for the Lagrangian formulation. The crack shape can be determined by methods based on various assumptions.

Any strict analysis must account for the Barenblatt condition at the tip. Barenblatt ${ }^{16-18}$ showed that, to avoid infinite stress at the crack tip, the fluid front cannot extend all the way to the tip. This requires a narrowing, pointed tip that prevents fluid penetration. Experimental results have confirmed that this analysis is correct. ${ }^{14}$

The exact shape of the crack at the tip and over the rest of its length is determined by the fluid pressure distribution. A detailed analysis of pressure distribution that accounts for the Barenblatt condition has been developed. For field applications requiring only engineering accuracy, however, the exact shape is not needed. Results of detailed analyses show that crack dimensions are not critically dependent on exact crack shape. For practical purposes, deriving an approximate shape based on simplifying assumptions is sufficient.

Here we assume that pressure in the crack is constant over its length. Under these conditions, Sneddon ${ }^{19}$ has shown that the crack shape is elliptic and is given by

$$
u_{y}(x, 0)=\frac{2\left(1-\nu^{2}\right) p_{e} L_{f}}{Y} \int_{L_{D}}^{1} \frac{\eta x(\eta) D \eta}{\sqrt{\eta^{2}-L_{D}^{2}}}
$$

where

$$
x(\eta)=\frac{2}{\pi} \int_{0}^{t} \frac{p(u) d \eta}{\sqrt{\eta^{2}-u^{2}}}
$$

In the Lagrangian analysis, this is consistent with setting $\gamma=\beta=\pi / 4$, which corresponds to an elliptic shape. For routine applications, this condition and the Sneddon shape give all the necessary accuracy.
Leakoff. We include fluid leakoff in the Lagrangian formulation by assuming that the flow rate can be divided into two parts: $q_{V}$, which contributes only to the fracture volume, and $q_{l}$, which contributes only to fluid loss through the fracture faces.

$$
q_{t}(x)=q_{V}(x)+q_{l}(x)
$$

$q_{V}(x)$ is given by $\mathrm{Eq} . \mathrm{A}-10$, and we take

$$
q_{l}(x)=2 \int_{x}^{L_{f}} v(x) \mathrm{d} x .
$$

We must distinguish between simple fracturing fluids, such as water and oil, and gelled fluids, which are wallbuilding. Simple fluids will be considered first.

We assume that leakoff occurs in accordance with Darcy's law with a piston-like displacement of the reservoir fluid by the fracture fluid filtrate. The filtrate is taken to be incompressible, and the reservoir fluid has compressibility $c$. The reservoir fluid is driven at a moving boundary that we designate $D_{i f f}(t)$.

Taking $W$ to be the fluid displacement and $\dot{W}$ as the flow rate per unit area, we have

$$
\dot{W}=\phi \dot{D}_{i f l} .
$$

In this notation, Darcy's law is written as

$$
v(t)=\dot{W}\left(D_{i f l}\right)=\frac{k}{\mu_{2}}\left(\frac{\partial p}{\partial y}\right)_{y=D_{i f f}} .
$$

From the theory of elasticity in porous media, we introduce the relative fluid displacement:

$$
\zeta=-\operatorname{div} W=-\frac{\partial W}{\partial y}=\frac{p}{M}+\alpha e .
$$

To simplify the leakoff problem, we take the compressibility of the rock matrix to be negligible compared with that of the pore fluid. We show in Appendix C that under these conditions,

$$
\zeta=c \phi p
$$

By analogy to heat flow, we assume that $\zeta$ can be represented by a parabolic approximation ${ }^{1}$ :

$$
\zeta=\zeta_{1}\left(1-\frac{y-D_{i f l}}{D_{c f l}}\right)^{2}
$$

We let the pressure at the moving boundary be $p_{1}=p\left(D_{i f l}\right)$.

These relationships are illustrated by the pressure diagram of Fig. 2. Appendix D shows how they lead to the following equation for the velocity of leakoff:

$$
v=\dot{W}=\sqrt{\frac{k \phi p_{e}}{2 \mu_{2}\left(t-t_{o}\right)}\left(\frac{\xi}{1+\xi}\right)}=\frac{\kappa}{\sqrt{t-t_{o}}} . \ldots
$$

Eq. 20 has the same form as the well-known relation for the velocity of leakoff given in the literature. ${ }^{20}$ 


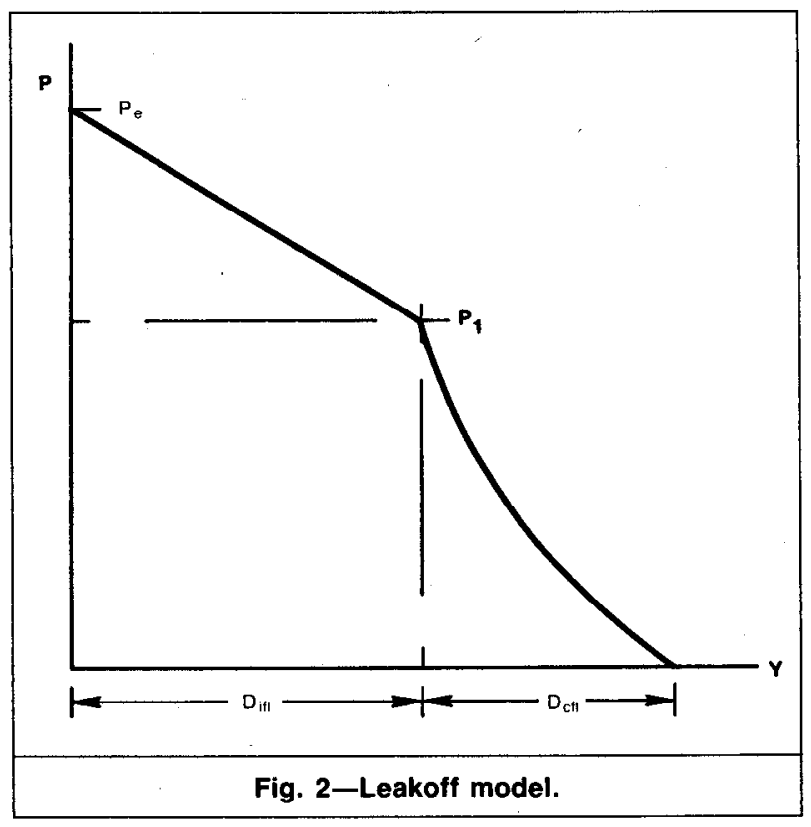

Although the form is the same, the coefficient $\kappa$ in Eq. 20 has a much different relation to fluid and reservoir properties than the constant $C_{t}$ in the literature. Furthermore, in practical cases, Eq. 20 gives a much higher rate of leakoff. Reasons for these differences are discussed in Appendix D.

For wall-building gelled fluids, we assume that the gel deposits a layer of low-permeability residue on the fracture face. As the wall forms, it produces a pressure drop $p_{w}-p_{e}$ across it. As a first approximation, we follow the usual assumption that there is no pressure drop until a certain volume of fluid, the spurt loss $W_{0}$, has leaked off; then the wall suddenly appears and begins to grow.

We assume that the pressure drop across the wall is proportional to the product of the leakoff rate and the volume of fluid that has passed through the wall. This gives

$$
p_{w}-p_{e}=0, W \leq W_{0}
$$

and

$$
p_{w}-p_{e}=\frac{1}{\mathcal{L}}\left(W-W_{0}\right) \dot{W}, W>W_{0}
$$

Thus the wall is assumed to grow in thickness throughout the leakoff period. Fig. 3 shows the pressure diagram for this case. Past the wall, it is no different from that in Fig. 2.

Appendix D shows that Eq. 21 leads to the velocity relation

$$
v=\dot{W}=\frac{\Lambda W_{0}+\sqrt{\Lambda^{2} W_{0}^{2}+8 t(1+\Lambda) \Lambda \Lambda p_{w}}}{4\left(t-t_{o}\right)(1+\Lambda)} \ldots
$$

for $W>W_{0}$. For $W \leq W_{0}$, the velocity is given by $\mathrm{Eq}$. 20. The constants $W_{0}$ and $\mathcal{A}$ must be determined experimentally. It can be shown that, for practical purposes,

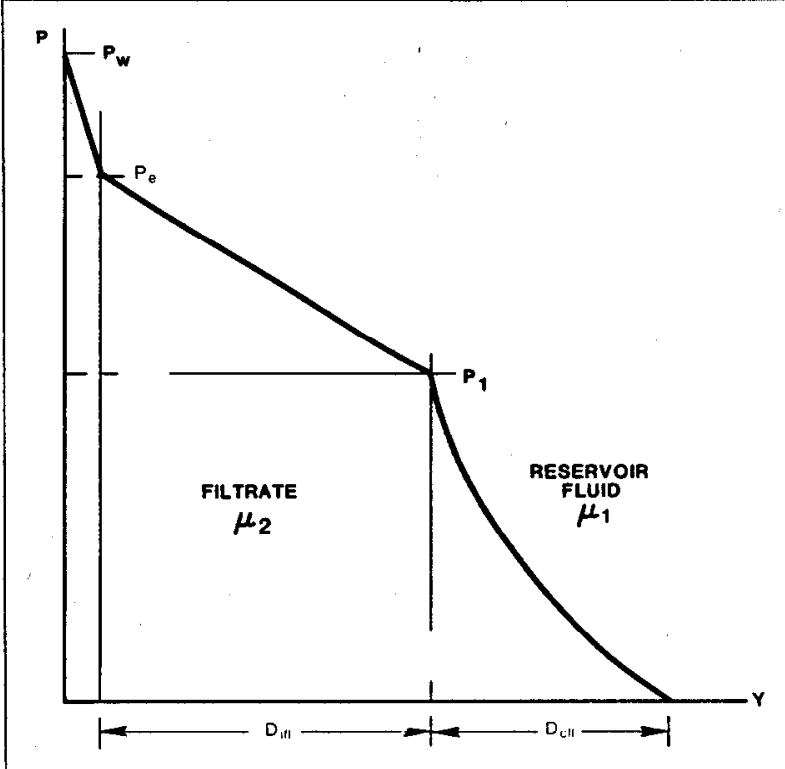

Fig. 3-Leakoff model with wall-building.

$W_{0}$ can be represented by the intercept and $\tau t$ by the slope of the straight line obtained at long time by plotting filtrate volume through thin wafers vs. $\sqrt{t}$. These constants are routinely provided by service companies for all common fracturing fluids.

\section{Lagrangian Equations With Leakoff}

To develop the Lagrangian equations with leakoff, we use a virtual work formulation. For convenience, let

$$
F_{i}=\frac{\partial D}{\partial \dot{q}_{i}}
$$

Substituting this relation into the Lagrangian equation (Eq. 3) and using Eq. A-5, we derive

$$
F_{1}=Q_{1}
$$

and

$$
F_{2}+2 K \gamma b_{f}=Q_{2}
$$

Multiplying Eq. 24 by $-L_{f}$ and Eq. 25 by $b_{f}$, adding the two, and using Eq. A-23, we obtain

$$
E L_{f}=F_{2} b_{f}-F_{1} L_{f}+2 K \gamma b_{f}^{2}
$$

In Appendix $\mathrm{F}$ we show how the virtual work concept can be used to find relations between $F_{1}, F_{2}$, and $L_{f}, b_{f}$, and to derive the important relation

$$
E-\frac{V_{0}}{\beta} \int_{0}^{1} \frac{\partial p}{\partial x} L_{D} \mathrm{f}\left(L_{D}\right) \mathrm{d} L_{D}=\frac{K \gamma}{2 L_{f}^{3}}\left(\frac{V_{0}}{\beta}\right)^{2} \ldots
$$

This is the hasic practical result of the I agrangian analysis. It can be solved numerically for $L_{f}$ when the pressure distribution $\partial p / \partial x$ is known. With $L_{f}$ the other variables $b_{f}$ and $p_{e}$ can be determined readily. 
The physics of the crack propagation problem is summarized concisely by Eq. 27 . It can be regarded as a simple energy balance expression. The first term is the separation energy already defined in terms of $\sigma$ and $\dot{\alpha}$. The second term is the energy associated with the generation of an internal pressure in the crack volume, accounting for leakoff. The sum of these terms represents work put into the process of crack propagation. It must equal the work done in producing the crack, which is the last term.

\section{Evaluation of Pressure Distribution}

To generalize the problem of determining $\partial p / \partial x$, we must consider both non-Newtonian and Newtonian fluids. For non-Newtonian fluids, we assume the common power-law relation.

$$
\dot{\epsilon}=K^{\prime} \tau^{n} \text {. }
$$

Newtonian fluids are represented by the same relation, with $n=1$ and $\mu=1 / K^{\prime}$. In our notation, $n$ is the inverse of $n^{\prime}$, the flow behavior index used by service companies, but $K^{\prime}$ is the same as their consistency index.

We consider flow between parallel fracture faces under the pressure gradient $\partial p / \partial x$. If we take the $x$ axis to be midway between the faces, the shear stress distribution across width $b$ satisfies the equilibrium condition.

$$
\frac{\partial \tau}{\partial y}=-\frac{\partial p}{\partial x}=a .
$$

This gives a shear stress distribution of

$$
\tau=a y \text {. }
$$

The fluid velocity $v$ has a profile of the type illustrated in Fig. 4. It satisfies the relation

$$
\dot{\epsilon}=-\frac{\partial v}{\partial y} \text {. }
$$

Integrating Eq. 31 gives the flow rate

$$
q_{t}=2 \int_{0}^{b / 2} v \mathrm{~d} y=\frac{K^{\prime} a^{n} b^{n+2}}{2^{n+1}(n+2)} .
$$

Solving for $a=-\partial p / \partial x$,

$$
-\frac{\partial p}{\partial x}=\left[\frac{2^{n+1}(n+2)}{K^{\prime} b^{n+2}} q_{t}\right]^{1 / n} \text {. }
$$

For Newtonian fluids with $n=1$, Eq. 33 reduces to Eq. A-8.

For convenience, we let

$$
R=\left[\frac{2^{n+1}(n+2)}{K^{\prime}}\right]^{1 / n} .
$$

From Eqs. 4, 34, A-14, and A-19, we obtain

$$
\frac{\partial p}{\partial x}=-R q_{t}^{1 / n}\left[\frac{\beta L_{f}}{V_{0} \mathrm{f}\left(L_{D}\right)}\right]^{n+2 / n} .
$$

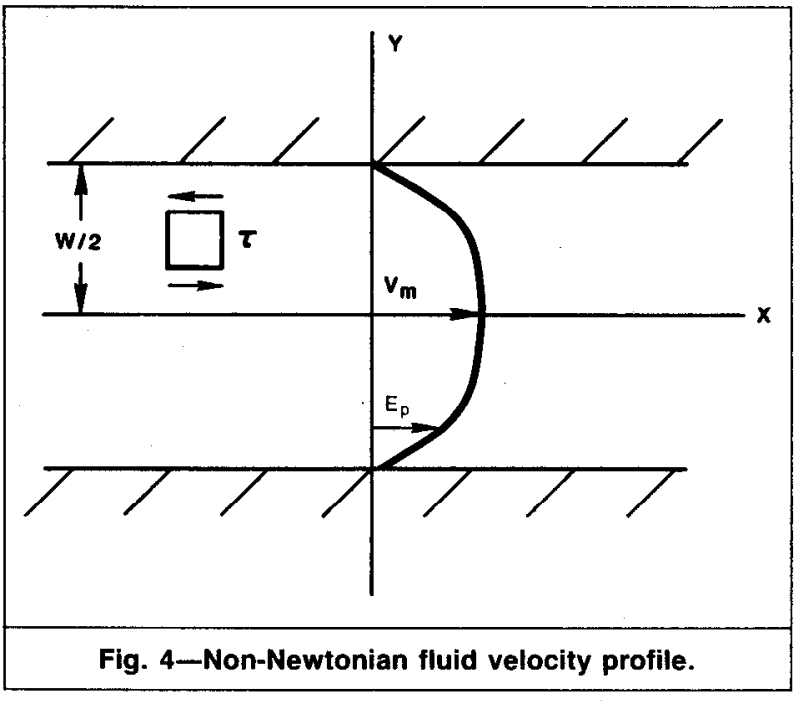

From Eqs. 13 and A-13, we obtain

$$
q_{t}=2\left(L_{f} \dot{b}_{f}+b_{f} \dot{L}_{f}\right) \mathrm{F}\left(L_{D}\right)+2 b_{f} \dot{L}_{f} L_{D} \mathrm{f}\left(L_{D}\right)+q_{l} .
$$

Differentiating Eq. A-9 to obtain $\dot{b}_{f}$ in Eq. 36 gives finally

$$
q_{t}=\frac{\mathrm{F}\left(L_{D}\right)}{\beta} \dot{V}_{0}+\frac{V_{0}}{\beta} L_{D} \mathrm{f}\left(L_{D}\right) \frac{\dot{L}_{f}}{L_{f}}+q_{l} .
$$

Eqs. 35 and 37 substituted in Eq. 27 give an equation in $L_{f}$ only. This equation can be solved for $L_{f}$ by straightforward iterative methods.

\section{Practical Evaluation}

We have developed a number of computer programs for solving Eq. 27 and determining $L_{f}, b_{f}$ and $p_{e}$ as functions of time at constant injection rate. The sophistication of these programs has been tailored to particular needs. For most routine field applications where only engineering accuracy is required, we use a program with several sirnplifying assumptions to reduce computer time. The most important of these are assuming the crack shape to be elliptical so that $\gamma=\beta$ and taking the plasticity coefficient $\alpha=0$. The $\alpha=0$ assumption must be made with care, but it has been found to be appropriate in many rescrvoirs. Measurements of $\alpha$ in reservoir rock and the effects of plasticity on fracture dimensions are the subject of a separate paper. ${ }^{21}$

We have shown by Eq. 8 that without leakoff the Lagrangian method gives power-law relations in $L_{f}, b_{f}$, and $p_{e}$ vs. time at constant injection rate. This is true to a very good approximation but with slightly different exponents when there is leakoff present. This is illustrated in Fig. 5 where $L_{f}, b_{f}$, and $p_{e}$ results obtained from solutions of Eq. 27 are shown for cases of very large leakoff rates with fluid efficiencies $\Sigma<0.02$.

For non-wall-building fluids, the exponents $h, m$, and $r$ in Eq. 8 decrease with increasing leakoff-i.e., decreasing $\Sigma$. For $\Sigma \rightarrow 0$, we find $h \rightarrow 1 / 2, m \rightarrow 1 / 4$, and $r \rightarrow-1 / 4$.

For wall-building fluids, Appendix D shows fluid loss through the wall to be the rate-controlling mechanism. Factors associated with the moving boundary $D_{i f l}(t)$ in 


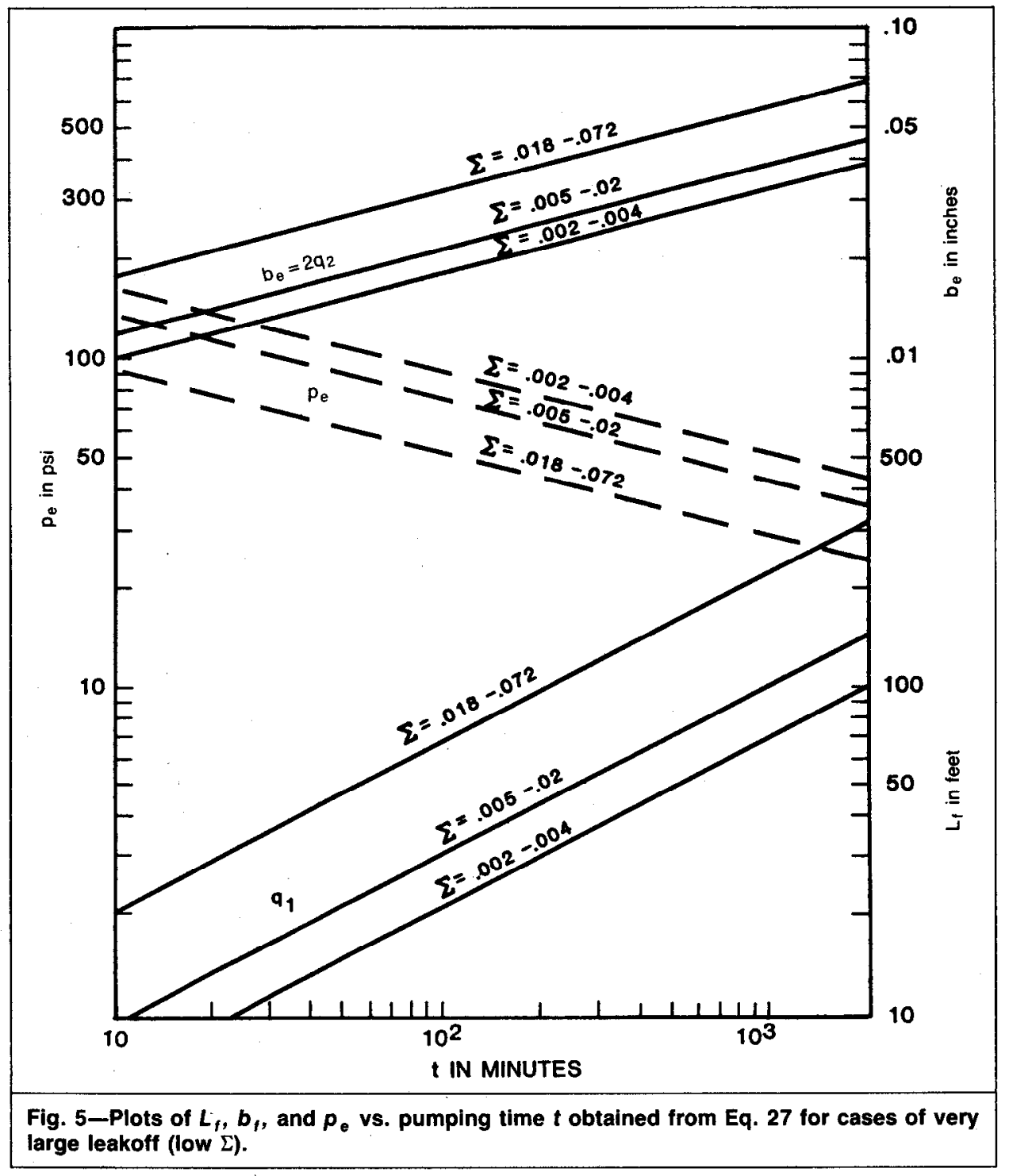

Fig. 3 are of little consequence. Fluid efficiencies are practically independent of fluid and reservoir properties apart from $\mathcal{A}$ and $W_{0}$.

These trends are very much in line with experimental data reported earlier from laboratory measurements of fracture propagation in small blocks. ${ }^{14}$ Although these experiments produced three-dimensional (3D) cracks, the results are surprisingly consistent with the $2 \mathrm{D}$ Lagrangian theory presented here.

We have made numerous comparisons between results obtained from our Lagrangian method with $\alpha=0$ and those obtained from other 2D theories. Experience from these comparisons has been compiled over a number of years for a great variety of cases. For $\sigma=0$ and with no leakoff, we find good agreement between the Lagrangian method and the methods of Zheltov and Khristianovich, ${ }^{4,5}$ Geertsma and de Klerk, ${ }^{8}$ and Daneshy. ${ }^{9}$ Significant discrepancies do not arise until $\sigma$ exceeds $1 \times 10^{5} \mathrm{erg} / \mathrm{cm}^{2}$ $\left[1 \times 10^{5} \mathrm{~mJ} / \mathrm{m}^{2}\right]$ and becomes large only when $E$ approaches $1 \times 10^{6} \mathrm{erg} / \mathrm{cm}^{2}\left[1 \times 10^{6} \mathrm{~mJ} / \mathrm{m}^{2}\right]$. The limited data compiled for $\sigma$ from laboratory measurements ${ }^{22}$ indicate that $\sigma$ does not exceed $1 \times 10^{5} \mathrm{erg} / \mathrm{cm}^{2}[1 \times$ $\left.10^{5} \mathrm{~mJ} / \mathrm{m}^{2}\right]$ in more than a few types of well-cemented reservoir rock. Thus, according to the Lagrangian anal- ysis, fracture propagation in most reservoirs is controlled mainly by the elastic energy required to force the fracture faces apart and not by surface energy effects at the tip. This is expected intuitively but has not been demonstrated convincingly before.

When leakoff without wall-building is included, the Lagrangian method gives much higher leakoff rates and significantly shorter fractures than the methods of Geertsma and de Klerk ${ }^{8}$ and Daneshy. ${ }^{9}$ This has been demonstrated in numerous comparisons with the Daneshy program for a variety of field cases. Although fewer comparisons have been done with the Geertsma and de Klerk method, it is reported to give results in general agreement with the Daneshy program. ${ }^{13}$

When wall-building is included with leakoff, the Lagrangian method gives results substantially in agreement with the Daneshy program. This agreement can be viewed as a natural consequence of points discussed earlier and of the similar methods of treating the wall-building process. In the Daneshy program, fluid loss is matched numerically to experimental data on leakoff through thin wafers. The same experimental data are used to obtain $\mathcal{A}$ and $W_{0}$ in the Lagrangian method. Because fluid loss through the wall dominates leakoff in this case, these 
methods should give about the same rate of leakoff. The major remaining difference between the two methods is the formulation used to combine fluid loss and crack growth. The substantial agreement between the two shows that both formulations lead to about the same result.

The Lagrangian method gives results much different from the Perkins and Kern equations ${ }^{6}$ and the associated Nordgren theory. ${ }^{7}$ The differences show most noticeably in the exponent $r$, which has an opposite sign in results computed by the two methods. These discrepancies are well-known from past comparisons of the PerkinsKern equations with $2 \mathrm{D}$ theories. They have been attributed to fundamental differences between $2 \mathrm{D}$ and $3 \mathrm{D}$ theorics. It should be noted, however, that laboratory experiments with 3D fracture propagation have agreed much better with 2D theories than with the Perkins and $\mathrm{Kern}^{6}$ or Nordgren ${ }^{7}$ theories. The most definitive test is in the sign of $r$, which is always negative in the laboratory experiments. ${ }^{14}$

The method used here of combining the Lagrangian formulation with a virtual work analysis provides a powerful means of treating the fracture propagation problem. The Lagrangian formulation can be used to separate the problem into its various parts. Each of these parts can be treated individually by completely analytic methods. Then, through the virtual work concept, they can be recombined into the central problem. Elastic deformation, separation energy, crack shape, fluid flow along the crack, and leakoff are all parts of the problem that have been treated in this way. All are recombined by the virtual work analysis in Eq. 27. The result is a largely analytic analysis that does not require the use of crude and uncertain finite element methods at any step.

\section{Fleld Applications}

The Lagrangian method described here has been used in various forms for the design of fracturing treatments for more than 10 years. Applications have ranged from simple estimates of fracture dimensions and fluid/sand volumes to sophisticated economic optimization programs for large fields with fracturing treatments in many wells. The method has also been extended to investigate fracture extension in waterflood operations. In most of these applications, the predicted fracture dimensions have been in line with production experience before and after fracturing except in unusual cases. Some of the more important field applications are listed in Table 1.

The Pembina application in Table 1 made use of smallvolume fracture treatments to improve waterflood operations in the Cardium oil sand. Hundreds of producing wells were fractured with a crosslinked gel carrying 12 to $15 \mathrm{lbm} / \mathrm{gal}$ [ 1438 to $1797 \mathrm{~kg} / \mathrm{m}^{3}$ ] sand at 20 to 30 $\mathrm{bbl} / \mathrm{min}\left[0.05\right.$ to $\left.0.08 \mathrm{~m}^{3} / \mathrm{s}\right]$. The main purpose was to provide communication over the complete Cardium sand interval. The Lagrangian program predicted no more than two-fold productivity increases caused by fracturing as opposed to four- to five-fold improvements forecast by service company programs. A 1.9-fold improvement was obtained as the average for more than 100 cases.

The Panoma/Council Grove application resulted in very large treatments carrying $2 \mathrm{lbm} / \mathrm{gal}\left[240 \mathrm{~kg} / \mathrm{m}^{3}\right]$ sand in slick water at rates from 70 to $150 \mathrm{bbl} / \mathrm{min}[0.19$ to 0.4 $\left.\mathrm{m}^{3} / \mathrm{s}\right]$. Early lreatments designed by service companies used only $25,000 \mathrm{lbm}[11340 \mathrm{~kg}$ ] of sand at $0.5 \mathrm{lbm} / \mathrm{gal}$
TABLE 1-SOME IMPORTANT FIELD APPLICATIONS OF LAGRANGIAN METHODS
Field

Pembina

Panoma/Council Grove

Canadian

Lasater

Various gulf coast wells

Tip-Top

Oldenburg

Piceance basin

South Belridge
Location

Alberta, Canada

Southwestern Kansas

Texas panhandle

East Texas

Texas

Midwestern Wyoming

West Germany

Western Colorado

Kern County, CA
[60 kg/m ${ }^{3}$ ]. Based on the Lagrangian method, an economic optimization program called for treatments in the $250,000-\mathrm{lbm}[11340-\mathrm{kg}]$ range at $2 \mathrm{lbm} / \mathrm{gal}$ and predicted dimensions that would give a six- to seven-fold improvement over the small treatments. The larger treatment was carried out in a well adjacent to a small treatment well and gave production results very consistent with this prediction. The large treatments have since been used successfully in hundreds of wells in this field.

The Canadian gas field application was very similar to the Panoma/Council Grove case. In early wells, service companies designed small treatments with less than 50,000 lbm [22 $680 \mathrm{~kg}]$ of sand to be carried in thin guar fluids at 20 to $30 \mathrm{bbl} / \mathrm{min}\left[0.05\right.$ to $0.08 \mathrm{~m}^{3} / \mathrm{s}$ ]. Based on the Lagrangian method, the treatment size was increased to $300,000 \mathrm{lbm}[136080 \mathrm{~kg}]$ at $3 \mathrm{lbm} / \mathrm{gal}\left[260 \mathrm{~kg} / \mathrm{m}^{3}\right]$ and 50 to $60 \mathrm{bbl} / \mathrm{min}\left[0.13\right.$ to $\left.0.16 \mathrm{~m}^{3} / \mathrm{s}\right]$. Following successful experience with this design in the first few wclls, the treatment size was increased in later wells to as much as $500,000 \mathrm{lbm}[226800 \mathrm{~kg}]$. The large treatments were used in more than 25 wells in the field with calculated absolute open flows ranging fom 8 to $60 \mathrm{MMcf} / \mathrm{D}$ $\left[227 \times 10^{3}\right.$ to $\left.1700 \times 10^{3} \mathrm{~m}^{3} / \mathrm{d}\right]$.

Experience in the Panoma/Council Grove and Canadian fields led to the concept of larger treatments in lowpermeability sands than had been considered practical before. Because of their routine success, these treatments served as forerunners of very large fracture treatments carried out in the Lasater and Tip Top fields and later in the Oldenburg and Piceance Creek fields, which are described elsewhere. ${ }^{23,24}$

The U.S. gulf coast wells included in Table 1 were selected from a variety of gas fields with completions in the Frio, Wilcox, and similar sands. These treatments were designed principally to remove skin damage evaluated from pressure buildup analyses in partially depleted wells. The Lagrangian method was used with an economic optimization program to determine treatment size on the basis of optimum fracture length beyond the skin damage.

In the South Belridge field, the Lagrangian method was applied to fracturing long, vertical, oil-producing intervals of diatomaceous earth. These treatments were designed to be near-massive on a vertical scale but modest on a fracture-length scale. Details of this work are discussed elsewhere. ${ }^{25}$

\section{Conclusions}

The Lagrangian method has been applied successfully to the problem of 2D fracture propagation. This method of analysis has introduced some new concepts and led to a 
practical method of calculating fracture dimensions for field operations.

The Lagrangian formulation provides a means of separating the crack propagation problem into parts that can be treated individually by largely analytic methods. These parts then can be recombined by mean of a virtual work analysis. Leakoff, fluid flow and pressure distribution along the crack, shape of the crack, elastic energy, and separation energy associated with crack growth can be treated in this way.

Leakoff has been considered as a piston-like displacement of compressible reservoir fluid by an incompressible fracture fluid filtrate in accordance with Darcy's law. This analysis, incorporated into the Lagrangian formulation, predicts much higher leakoff rates than conventional methods, provided the fluid is not wall-building. For wall-building fluids, leakoff through the wall is the ratelimiting step. In this case the Lagrangian method predicts fluid loss rates that agree with conventional methods based on spurt loss and long-time rate of filtration through thin wafers.

Associated with fracture propagation is an elastic deformation energy and a separation energy. The latter is almost negligible unless the Griffith surface tension is large or unless there is significant plastic deformation. The surface tension must exceed $1 \times 10^{5} \mathrm{erg} / \mathrm{cm}^{2}$ $\left[1 \times 10^{5} \mathrm{~mJ} / \mathrm{m}^{2}\right]$ to be important. On the basis of limited data, this appears to be uncommon in reservoir rock. Therefore, excluding cases of plastic behavior, fracture propagation is controlled mainly by the elastic energy required to force the fracture walls apart and has little to do with surface energy effects at the tip.

In the absence of leakoff, the Lagrangian equations predict power-law relations in fracture length $L_{f}$, fracture half-width $b_{f}$, and injection pressure $p_{e}$ vs. time. The power-law exponents are $h=2 / 3$ for $L_{f}, n=1 / 3$ for $b_{f}$, and $r=-1 / 3$ for $p_{e}$. When leakoff does exist, power-law relations are still found to a good approximation, but the exponents change with fluid efficiency $\Sigma$. As $\Sigma \rightarrow 0$, $m \rightarrow 1 / 2, n \rightarrow 1 / 4$, and $r \rightarrow-1 / 4$. These trends agree with laboratory results obtained in 3D fracturing experiments.

Field applications of the Lagrangian method have generally predicted fracture dimensions consistent with production data except in unusual cases.

\section{Nomenclature}

$a=$ notation used for convenience, defined by Eq. 29

$\mathcal{A}=$ proportionality constant for wall-building fluid defined by Eq. 21

$A, B, C=$ constants identified by Eq. A-16

$b=$ fracture width, function of $x$ and $t$

$b_{e}=$ fracture width at entrance, function of $t$ only

$b_{f}=$ fracture half-width at entrance

$C_{1}, C_{2}=$ compressibility of reservoir fluid

$C_{3}, C_{4}=$ constants appearing in Solutions 8 to Lagrangian Eqs. 6 and 7 for cases of no leakoff

$G_{1}=$ constant associated with velocity of filtration into empty pores, defined by Eq. D-22
$\mathscr{G}_{2}=$ constant associated with velocity of fluid flow resisted by compression of reservoir fluid, defined by Eq. D-23

$G_{1}=$ composite fluid loss coefficient obtained from $\mathscr{G}_{1}$ and $\mathscr{G}_{2}$ by Eq. D-24

$D=$ dissipation function

$D_{c f l}=$ penetration depth associated with compression of reservoir fluid by fracture fluid front illustrated in Fig. 3

$D_{i f l}=$ penetration depth of incompressible fracture fluid filtrate beyond fracture face illustrated in Fig. 3

$e=$ dilatation

$E=$ separation energy, defined by Eq. A-21

$E_{k}=$ kinetic energy of system

$E_{p}=$ potential energy of system

$\mathrm{f}\left(L_{D}\right)=$ shape function, defined by Eq. 4

$F_{i}=$ notation used for convenience in virtual work analysis, defined by Eq. 23

$\mathrm{F}\left(L_{D}\right)=$ shape function, detined by Eq. A-11

$G=$ shear modulus

$\mathscr{G}=$ notation used for convenience, defined by Eq. B-5

$h=$ exponent appearing in Solutions 8 to Lagrangian Eqs. 6 and 7 for no leakoff

$H=$ notation used for convenience, defined by Eq. B-6

$k=$ permeability of reservoir rock

$K=$ elastic constant defined by Eq. A-3

$K^{\prime}=$ constant in power-law relation assumed for non-Newtonian fluids in Eq. 28

$L=$ Lagrangian function given by difference between kinetic and potential energy of system

$L_{D}=$ dimensionless distance along crack

$L_{f}=$ fracture half-length from wellbore to tip

$m=$ exponent appearing in Solutions 8 to

Lagrangian Eqs. 6 and 7 for no leakoff

$M=$ poroelastic coefficient defined in Ref. 21

$M_{c}=$ poroelastic coefficient defined by Eq. C-5

$n=$ exponent in power-law relation assumed for non-Newtonian fluids in Eq. 28

$n^{\prime}=$ flow behavior index, the inverse of $n$

$p=$ fluid pressure in fracture as function of $x$

$p_{e}=$ fluid pressure at fracture entrance

$p_{w}=$ fluid pressure impinging on wall formed by wall-building fluid

$p_{\infty}=$ far-field stress

$p_{1}=$ fluid pressure at moving boundary between fracture fluid filtrate and reservoir fluid as illustrated in Fig. 3

$q_{i}=$ generalized coordinates for system

$q_{l}=$ portion of $q_{t}$ contributing to leakoff only

$q_{t}=$ total flow rate in fracture, function of $x, t$

$q_{t e}=$ total flow rate into fracture entrance; function of $t$ only

$q_{V}=$ portion of $q_{t}$ contributing to fracture volume only 
$Q_{i}=\bar{Q}_{i}$ forces not derived from a dissipation function

$\bar{Q}_{i}=$ forces not derived from a potential function

$r, s=$ exponents appearing in Solutions 8 to Lagrangian Eqs. 6 and 7 for no leakoff

$R=$ notation used for convenience, defined by Eq. 34

$t=$ time variable

$t_{0}=$ time when crack reaches Point $x$

$u=$ displacement as function of $\mathrm{x}$

$\bar{u}=$ rock matrix displacement vector

$u_{y}=$ displacement as function of $x$ at $y=0$

$v=$ fluid velocity, function of $x$ only for

Newtonian fluid without leakoff;

function of $x$ and $y$ for non-Newtonian

fluid or for leakoff

$V=$ volume of fracture from $x$ to $L_{f}$

$V_{0}=$ volume of fracture from entrance to $L_{f}$

$W=$ fluid displacement associated with leakoff

$W_{0}=$ spurt loss for wall-building fluid

$\mathscr{W}=$ virtual work associated with generalized forces $Q_{i}$

$\mathscr{W}_{c}=$ portion of $\mathscr{H}^{\prime}$ caused by extending the crack

$\mathscr{W}_{p}=$ portion of $\mathscr{W}$ done by fluid pressure in increasing crack volume

$x, y, z=$ Cartesian coordinates oriented as shown in Fig. 1

$Y=$ Young's modulus

$Z=$ constant defined by Eq. B-4

$\alpha=$ poroelastic constant, defined in Ref. 27

$\beta=$ shape constant, defined by Eq. A-14

$\gamma=$ shape constant, defined by Eq. A-6

$\Gamma=$ plasticity coefficient, defined by Eq. A-21

$\dot{\epsilon}=$ shear rate in non-Newtonian fluid

$\zeta=$ relative fluid displacement, defined by Fq. 17

$\zeta_{1}=$ value of $\zeta$ at moving boundary; $y=D_{i f l}$ used in parabolic approximation, Eq. 19

$\eta=$ dummy variable used in Sneddon Eq. C-12

$\kappa=$ coefficient associated with velocity of leakoff defined by Eq. 20

$\lambda=$ Lamé constant

$\Lambda=$ notation used for convenience, defined by Eq. D-26

$\mu=$ viscosity of Newtonian fluid in fracture

$\mu_{1}=$ viscosity of reservoir fluid ahead of moving boundary $D_{i f l}(t)$ as in Fig. 3

$\mu_{2}=$ viscosity of fracture fluid filtrate behind moving boundary $D_{i f l}(t)$ as in Fig. 3

$\nu=$ Poisson's ratio

$\xi=$ notation used for convenience, defined by Eq. D-18

$\sigma=$ surface tension associated with brittle crack propagation as originally defined by Griffith

$$
\begin{aligned}
\Sigma & =\text { fluid efficiency given by } q_{v} /\left(q_{\nu}+q_{l}\right) \\
\tau & =\text { shear stress in non-Newtonian fluid } \\
\phi & =\text { porosity of reservoir rock } \\
\chi & =\text { dummy variable in Sneddon Eq. } 12 \\
\psi & =\text { shape function defined by Eq. A-2 }
\end{aligned}
$$

\section{Acknowledgments}

We wish to thank R.E. Aikin and A.B. Craig for field and laboratory assistance; E.L. Cook, J.L. Fitch, T.C. Vogt, and M.K. Strubhar for numerous contributions in discussions; and Mobil Research and Development Corp. for permission to publish this paper.

\section{References}

1. Biot, M.A.: "New Variational Lagrangian Irreversible Thermodynamics with Application to Viscous Flow; ReactionDiffusion and Solid Mechanics," Advances in Applied Mechanics, Academic Press, New York City (1984) 24, 1-91.

2. Biot, M.A.: Variational Principles in Heat Transfer, Oxford Press, London (1970).

3. Biot, M. A.: "Non-Linear Effect of Initial Stress in Crack Propa gation Between Similar and Dissimilar Orthotropic Media," Applied Math. Quart. (1970) 30, 379-406.

4. Zheltov, Y.P. and Khristianovitch, S.A.: "Hydraulic Fracture of an Oil-Bearing Bed," Izvestia Akademii Nauk S.S.S.R., OTN (1955) No. 5, 3-41.

5. Khristianovitch, S.A. and Zheltov, Y.P.: "Formation of Vertical Fractures by Means of Highly Viscous Liquid,' Proc., Fourth World Pet. Cong., Rome (1955) Sec. II, 579-86.

6. Perkins, T.K. and Kern, L.R.: "Widths of Hydraulic Fractures,", J. Pet. Tech. (Sept. 1961) 937-49; Trans., AIME, 222.

7. Nordgren, R.P.: "Propagation of a Vertical Hydraulic Fracture," Soc. Pet. Eng. J. (Aug. 1972) 306-14; Trans., AIME, 253.

8. Geertsma, J. and de Klerk, F.: "A Rapid Method of Predicting Fracture Width and Extent of Hydraulically Induced Fractures," J. Pet. Tech. (Dec. 1969) 1571-81; Trans., AIME, 246.

9. Daneshy, A.A.: "On the Design of Vertical Hydraulic Fractures," J. Pet. Tech. (Jan. 1973) 83-97; Trans., AIME, 255.

10. Le Tirant, P. and Dupuis, M.: "Dimensions of the Fractures Obtained by Hydraulic Fracturing of Oil Bearing Formations,"' Rev. Inst. Franc, Pet. (Jan. 1967) 44-98.

11. Cleary, M.P.: "Rate and Structure Sensitivity in Hydraulic Fracturing of Fluid Saturatcd Porous Formations,'” Proc., 1979 U.S. Rock Mech. Symp., U. of Texas, Austin (June) 127-42.

12. Cleary, M.P.: "Comprehensive Design Formulae for Hydraulic Fracturing," paper SPE 9259 presented at the 1980 SPE Annual Technical Conference and Exhibition, Dallas, Sept. 21-24.

13. Geertsma, J. and Haafkens, R.: "A Comparison of the Theories for Predicting Width and Extent of Vertical Hydraulically Induced Fractures," J. Eng. Res. Tech. (March 1979) 101, 8-19.

14. Medlin, W.L. and Massé, L.: "Laboratory Experiments in Fracture Propagation," Soc. Pet. Eng. J. (June 1984) 256-68.

15. Flugge, W.: Handbook of Engineering Mechanics, McGraw-Hill Book Co. Inc., New York City (1962) 23-35.

16. Barenblatt, G.I.: "On Certain Problems of the Theory of Elasticity that Arise in the Investigation of the Mechanism of Hydraulic Rupture of an Oil-Bearing Layer," Prikladnaia Matematika $i$ Mehanika Akademii Nauk S.S.S.R. (1956) 20, 475-86.

17. Barenblatt, G.I.: "An Approximate Evaluation of the Size of a Crack Forming in Hydraulic Fracture of a Stratum," Izvestia Akademii Nauk S.S.S.R., OTN (1957) No. 3, 1980-82.

18. Barenblatt, G.I.: "Equilibrium Cracks Forming During Brittle Fracture," Dokladi Akademii Nauk S.S.S.R. (1959) 127, 47-50.

19. Sneddon, I.N. and Lowengrud, M.: Crack Problems in the Classical Theory of Elasticity, J. Wiley \& Sons, Inc., New York City (1969) 28.

20. Howard, G.C. and Fast, C.R.: Hydraulic Fracturing, Monograph Series, SPE, Richardson, TX (1970) 2, 34.

21. Mẹllin, W.L. and Massé, L.: "Plasticity Effects in Hydraulic Fracturing," paper SPE 11068 presented at the 1982 SPE Annual Technical Conference and Exhibition, New Orleans, Sept. 26-29.

22. Perkins, T.K. and Krech, W.W.: "Effect of Cleavage Rate and Stress Level on Apparent Surface Energies of Rock," Soc. Pet. Eng. J. (Dec. 1966) 308-14; Trans., AIME, 237. 
23. Slusser, M.L. and Rieckmann, M.: "Fracturing Low Permeable Gas Reservoirs," Erdoel-Erdgas Zeitschrift (March 1976) 92.

24. Strubhar, M.K., Fitch, J.L., and Medlin, W.L.: "Demonstration of Massive Hydraulic Fracturing, Piceance Basin, Colorado," paper SPE 9336 presented at the 1980 SPE Annual Technical Conference and Exhibition, Dallas, Sept. 21-24.

25. Strubhar, M.K. et al.: "Fracturing Results in Diatomaceous Earth Formations, South Belridge Field, California," J. Pet. Tech. (March 1984) 495-502.

26. Mathews, J. and Walker, R.L.: Mathematical Methods of Physics, W.A. Benjamin Inc., New York City (1965) 73.

27. Biot, M.A.: "Mechanics of Deformation and Acoustic Propagatimn in Porous Media," J. Appl. Phys. (April 1962) 33, 1482-98.

28. Williams, B.B.: "Fluid Loss from Hydraulically Induced Fractures," J. Pet. Tech. (July 1970) 882-88; Trans., AIME, 249.

\section{Appendix A}

We consider the crack of Fig. 1 for the case of a Newtonian fracture fluid without leakoff. The elastic potential energy in the half-space $x>0$ is

$$
E_{p}=\int_{0}^{L_{f}} \int_{0}^{b} p \mathrm{~d} b \mathrm{~d} x
$$

From elasticity theory, the pressure, $p$, can be written as

$$
p=K \frac{b_{f}}{L_{f}} \psi\left(L_{D}\right)
$$

where $\psi$ depends on the shape of the crack. For simplicity, we assume the shape is elliptical. In this case,

$$
K=\frac{Y}{2\left(1-\nu^{2}\right)}
$$

and

$$
\psi\left(L_{D}\right)=1
$$

Substituting Eqs. 4, A-2, A-3, and A-4 into Eq. A-1 yields

$$
E_{p}=K \gamma b_{f}^{2}
$$

with

$$
\gamma=\int_{0}^{1} \mathrm{f}\left(L_{D}\right) \psi\left(L_{D}\right) \mathrm{d} L_{D} .
$$

The dissipation function is half the power dissipated over the crack length.

$$
D=-1 / 2 \int_{0}^{L_{f}} q_{t}(x) \frac{\partial p}{\partial x} \mathrm{~d} x
$$

We assume Poiseuille flow in the fracture, so

$$
q_{f}(x)=-\frac{b^{3}}{12 \mu} \frac{\partial p}{\partial x}
$$

Substituting Eq. A-8 into A-7 gives

$$
D=6 \mu \int_{0}^{L_{f}} \frac{q_{t}^{2}(x)}{b^{3}} \mathrm{~d} x .
$$

To express this result in terms of the Lagrangian coordinates, $q_{i}$ and $\dot{q}_{i}$, we express $q_{t}$ in terms of the time derivative of the crack volume.

$$
q_{t}(x)=\dot{V}=2 \frac{\mathrm{d}}{\mathrm{d}_{t}} \int_{x}^{L_{f}} b \mathrm{~d} x .
$$

Substituting Eq. 4 and letting

$$
\mathrm{F}\left(L_{D}\right)=\int_{L_{D}}^{1} \mathrm{f}\left(L_{D}\right) \mathrm{d} L_{D}
$$

gives

$$
\begin{aligned}
q_{1}(x) & =2\left(L_{f} \dot{b}_{f}+b_{f} \dot{L}_{f}\right) \mathrm{F}\left(L_{D}\right) \\
& +b_{f} \dot{L}_{f} L_{D} \mathrm{f}\left(L_{D}\right), \ldots
\end{aligned}
$$

or at the crack entrance,

$$
q_{l}(0)=q_{t e}=2 \beta\left(L_{f} b_{f}+b_{f} \dot{L}_{f}\right),
$$

where

$$
\beta=\mathrm{F}(0)=\int_{0}^{1} \mathrm{f}\left(L_{D}\right) \mathrm{d} L_{D} .
$$

Substituting Eqs. A-12 and 4 into Eq. A-9 gives the needed expression for the dissipation function.

$$
\begin{aligned}
D & =\frac{3 \mu L_{f}}{b_{f}{ }^{3}}\left[A\left(L_{f} \dot{b}_{f}+b_{f} \dot{L}_{f}\right)^{2}\right. \\
& \left.+2 B\left(L_{f} \dot{b}_{f}+b_{f} \dot{L}_{f}\right) b_{f} \dot{L}_{f}+C b_{f}^{2} \dot{L}_{f}^{2}\right]
\end{aligned}
$$

where

$$
\begin{aligned}
& A=\int_{0}^{1} \frac{\mathrm{F}^{2}\left(L_{D}\right)}{f^{3}\left(L_{D}\right)} \mathrm{d} L_{D}, \\
& B=\int_{0}^{1} \frac{L_{D} \mathrm{~F}\left(L_{D}\right)}{\mathrm{f}^{2}\left(L_{D}\right)} \mathrm{d} L_{D},
\end{aligned}
$$

and

$$
C=\int_{0}^{1} \frac{L_{D}^{2}}{\mathrm{f}\left(L_{D}\right)} \mathrm{d} L_{D} .
$$

The generalized forces $Q_{i}$ can be derived from the virtual-work principle. A virtual work associated with these forces can be written as

$$
\mathrm{d} \mathscr{H}=Q_{1} \mathrm{~d} L_{f}+Q_{2} \mathrm{~d} b_{f} .
$$


This virtual work can be separated into two parts: that done by the fluid pressure in increasing the crack volume, $\mathscr{W}_{p}^{\prime}$, and that done in extending the crack, $\mathscr{W}_{c}$. We have

$$
\mathrm{d} \mathscr{I}_{p}^{\prime}=p_{e} \mathrm{~d} V_{0},
$$

with

$$
\begin{aligned}
V_{0}=q_{t e} t & =\int_{0}^{L_{f}} b \mathrm{~d} x=2 L_{f} b_{f} \int_{0}^{l} \mathrm{f}\left(L_{D}\right) \mathrm{d} L_{D} \\
& =2 \beta L_{f} b_{f}, \ldots \ldots \ldots \ldots \ldots
\end{aligned}
$$

where we have used Eq. 4 and Eq. A-14. Substituting Eq. A-19 into Eq. A-18 gives

$$
\begin{aligned}
& \mathrm{d} \mathscr{H}_{p}=2 \beta p_{e}\left(b_{f} \mathrm{~d} L_{f}+L_{f} \mathrm{~d} b_{f}\right) . . \\
& \mathrm{d} \mathscr{H}_{c}^{\prime}=-E \mathrm{~d} L_{f}=-(2 \sigma+\Gamma) \mathrm{d} L_{f},
\end{aligned}
$$

where $E$ is a separation energy associated with the Griffith surface tension $\sigma$ and a constant $\Gamma$ that accounts for plastic deformation of the reservoir rock, acoustic radiation, etc.

The virtual work associated with $Q_{1}$ and $Q_{2}$ can now be written

$\mathrm{d} \mathscr{I}^{\prime}=\left(2 \beta p_{c} b_{f}-E\right) \mathrm{d} L_{f}+2 \beta p_{c} L_{f} \mathrm{~d} b_{f}$.

Comparing Eq. A-23 with Eq. A-17 shows that

$$
Q_{1}=2 \beta p_{e} b_{f}-E
$$

and

$$
Q_{2}=2 \beta p_{\iota} L_{f} .
$$

\section{Appendix B}

To evaluate the constants $C_{1}, C_{2}$, and $C_{3}$ in Eq. 9, we begin by integrating Eq. A-13.

$$
q_{c c} t=2 \beta \int_{0}^{t}\left(L_{f} \dot{b}_{f}+b_{f} \dot{L}_{f}\right) \mathrm{d} t=2 \beta L_{f} b_{f}
$$

Using Eq. 9, we get

$$
C_{1} C_{2}=\frac{q_{t e}}{2 \beta}
$$

Substituting Eq. 9 into Eqs. 6 and 7 and eliminating $p_{a}$ yields

$$
\frac{\mu q_{r e}}{\beta}(3 B+2 C) \frac{C_{1} C_{2}}{C_{3}{ }^{2}}+E=2 K \gamma \frac{C_{2}{ }^{3}}{C_{1} C_{2}} .
$$

We let

$$
Z=\frac{C_{2}{ }^{3}}{C_{1} C_{2}},
$$

$$
c=\frac{3 B+2 C}{\beta \gamma}\left(\frac{\mu q_{t e}}{2 K}\right),
$$

and

$$
H=\frac{E}{2 K \gamma} .
$$

Making these substitutions in Eq. B-3 and solving for $Z$, we derive

$$
Z=1 / 2\left(H+\sqrt{H^{2}+4 \cdot \dot{\zeta}}\right) .
$$

Eqs. B-2 and B-4 give

$$
C_{1}=\left[\left(\frac{q_{t e}}{2 \beta}\right)^{2} \frac{1}{Z}\right]^{1 / 3}
$$

and

$$
C_{2}=\left(\frac{q_{1 c} Z}{2 \beta}\right)^{1 / 3}
$$

Substituting Eq. 9 into Eq. 7 and using Eqs. B-8 and B-9, we obtain

$$
\begin{aligned}
C_{3} & =\frac{K \gamma}{\beta}\left(\frac{q_{t e}}{2 \beta}\right)^{-1 / 3} \mathrm{Z}^{2 / 3} \\
& +\frac{3 \mu}{\beta}\left(A+\frac{2}{3} B\right)\left(\frac{q_{t e}}{2 \beta}\right) Z^{2 / 3} Z^{-4 / 3}
\end{aligned}
$$

As a practical illustration of these results, we show that conditions can be chosen for which the Lagrangian formulation gives the width equation obtained by Geertsma and de Klerk ${ }^{8}$ for no leakoff. We take $E=0$, which reduces $\mathrm{Eq}$. B-7 to

$$
Z=\sqrt{\zeta^{\prime}}
$$

making Eq. 10 valid. For an elliptical crack,

$$
f\left(L_{D}\right)=\sqrt{1-L_{D}^{2}} \text {. }
$$

$K$ and $\psi\left(L_{D}\right)$ are then given by Eqs. A-3 and A-4, and $\gamma=\beta=\pi / 4$. Letting $L_{D}=\cos \theta$, we derive from Eq. A-16

$$
B=\frac{1}{2} \int_{0}^{\pi / 2} \frac{(\theta-\sin \theta \cos \theta) \cos \theta}{\sin \theta} \mathrm{d} \theta=0.152
$$

and

$$
C=\int_{0}^{\pi / 2} \cos ^{2} \theta \mathrm{d} \theta=0.785
$$

Substituting these results into the second part of Eq. 10 and taking $\nu=0.25$, we obtain

$$
b_{c}=2 b_{f}=2.65 \sqrt[4]{\frac{\mu q_{t c} L_{f}^{2}}{Y}}=2.1 \sqrt[4]{\frac{\mu q_{t c} L_{f}^{2}}{G}} \ldots
$$


This result agrees with the width equation given by Geertsma and de Klerk ${ }^{8}$ for unit fracture height and is limited to cases in which the separation energy, $E$, is small. For reservoir rocks with significant surface energy, $\sigma$, it becomes a poor approximation.

\section{Appendix C}

From the theory of elasticity in porous media ${ }^{27}$ we make use of the following relations:

$$
\begin{aligned}
& G \nabla^{2} u+\left(G+\lambda+\alpha^{2} M\right) \operatorname{grad} e=\alpha \\
& \frac{\partial \zeta}{\partial t}=\frac{k M}{\mu}\left(\nabla^{2} \zeta-\alpha \nabla^{2} e\right), \ldots \ldots \\
& \left(2 G+\lambda+\alpha^{2} M \nabla^{2} e=\alpha M\right) \nabla^{2} \zeta, \\
& \frac{\partial \zeta}{\partial t}=\frac{k M_{c}}{\mu} \nabla^{2} \zeta \ldots \ldots \ldots \ldots \\
& M=\frac{1}{\phi c-\phi K(1-\alpha)-\alpha K(1-\alpha)},
\end{aligned}
$$

and

$$
M_{c}=\frac{M(\lambda+2 G)}{\lambda+2 G+\alpha^{2} M} \text {. }
$$

Substituting Eq. 17 into Eq. C-4 gives

$$
G \nabla^{2} \bar{u}+(\lambda+G) \operatorname{grad} e=\alpha \operatorname{grad} p .
$$

Integration of this equation gives

$$
G \operatorname{div} \bar{u}+(\lambda+G) e=\alpha p,
$$

where the constant of integration has been absorbed into $p$.

Eq. C-7 reduces to

$$
(\lambda+2 G) e=\alpha p .
$$

Substituting Eq. 17 again gives

$$
(\lambda+2 G)(M \zeta-p)=\alpha^{2} M p
$$

Solving for $p$ and using Eq. C-5 yields

$$
p=M_{c} \zeta \text {. }
$$

We assume the compressibility of the matrix to be negligible, so

$$
K \approx 0 \text {. }
$$

Substituting Eq. C-12 into Eqs. C-5 and C-6 gives

$$
M=M_{c}=\frac{1}{\phi c} \text {. }
$$

Substituting Eq: C-13 into Eq. C-11 gives Eq. 18.

\section{Appendix D}

Substituting the parabolic approximation (Eq. 18) into Eq. 17 and integrating yiclds

$$
W=\frac{1}{3} \zeta\left(D_{c f l}+D_{i f l}-y\right)
$$

At $y=D_{i f l}$, we obtain

$$
\dot{W}\left(D_{i f l}\right)=\frac{1}{3}\left[D_{c f l} \dot{\zeta}\left(D_{i f l}\right)+\left(\dot{D}_{c f l}+\dot{D}_{i f l}\right) \zeta\left(D_{i f l}\right)\right] .
$$

From Eq. 18,

$$
\dot{\zeta}\left(D_{i f l}\right)=\dot{\zeta}_{1}\left(D_{i f l}\right)+\frac{2 \dot{D}_{i f l}}{D_{c f l}} \zeta_{1}\left(D_{i f l}\right) .
$$

We also have, from Eq. 17,

$$
\dot{\zeta}=c \phi \dot{p} .
$$

Using Eqs. D-4, D-3, and 17 in Eq. D-2 gives

$$
\dot{W}\left(D_{i f l}\right)=\frac{1}{3} c \phi\left(D_{c f l} \dot{p}_{1}+3 p_{1} \dot{D}_{i f l}+p_{1} \dot{D}_{c f l}\right) \ldots
$$

At $y=D_{i f l}$, Eqs. 18 and 19 give

$$
\left(\frac{\partial p}{\partial y}\right)_{y=D_{i / l}}=-\frac{2 p_{1}}{D_{c f l}} .
$$

From Eq. 16, Darcy's law reduces to

$$
\dot{W}\left(D_{i f l}\right)=\frac{2 k p_{1}}{\mu_{1} D_{c f l}} .
$$

Equating Eqs. D-5 and D-7, we obtain

$$
c \phi\left(D_{c f l} \dot{p}_{1}+3 p_{1} \dot{D}_{i f l}+p_{1} \dot{D}_{c f l}\right)=\frac{6 k p_{1}}{\mu_{1} D_{c f l}}
$$

Darcy's law for the filtration process is

$$
\dot{W}\left(D_{i f l}\right)=\frac{k\left(p_{e}-p_{1}\right)}{\mu_{2} D_{i f l}} .
$$

Equating Eqs. D-9 and 15 gives

$$
\frac{k}{\mu_{2}}\left(p_{c}-p_{1}\right)=\phi D_{i f l} \dot{D}_{i f l},
$$

and equating Eqs. D-7 and 15 gives

$$
\frac{2 k}{\mu_{1}} p_{1}=\phi D_{c f l} \dot{D}_{i f l}
$$


Eqs. D-8, D-10, and D-11 provide three differential equations in $\dot{D}_{i f l}, \dot{D}_{c f}$, and $p_{1}$, which must be solved for $\dot{D}_{i f l}$. Eqs. D-10 and D-11 reduce to

$$
\frac{D_{i f l}}{D_{c f l}}=\frac{\mu_{1}\left(p_{e}-p_{1}\right)}{2 \mu_{2} p_{1}},
$$

which, by differentiation, gives

$$
\begin{aligned}
\left(\frac{2 D_{i f f}}{\mu_{1}}\right. & \left.+\frac{D_{c f l}}{\mu_{2}}\right) \dot{p}_{1}+\frac{2 p_{1} \dot{D}_{i f l}}{\mu_{1}}-\frac{\left(p_{e}-p_{1}\right) \dot{D}_{c f l}}{\mu_{2}} \\
& =\frac{D_{c f} \dot{p}_{e}}{\mu_{2}} \cdot \ldots \ldots \ldots \ldots \ldots \ldots \ldots \ldots
\end{aligned}
$$

Combining Eq. D-13 with Eqs. D-11 and D-8, we obtain the differential equations

$$
\begin{gathered}
\dot{D}_{i f l}=\frac{2 k p_{1}}{\phi D_{c f l}}, \ldots \ldots \ldots \ldots \ldots \ldots \ldots \ldots \ldots(\mathrm{D}-14) \\
\dot{p}_{1}= \\
\frac{p_{1}\left[\frac{6 k}{\mu_{1} \mu_{2}}\left(1-c p_{1}\right)\left(p_{e}-p_{1}\right)+c\left(\frac{\phi}{\mu_{2}} D_{c f l}{ }^{2} \dot{p}_{e}-\frac{4 p_{1}{ }^{2}}{\mu_{2}}\right)\right]}{c \phi D_{c f l}\left(\frac{2 D_{i f f} p_{1}}{\mu_{1}}+\frac{D_{c f f} p_{e}}{\mu_{2}}\right)},
\end{gathered}
$$

and

$\dot{D}_{c f l}=$

$$
\frac{\frac{6 p_{1}}{\mu_{1}}\left(\frac{2 k D_{i f f}}{\mu_{1}}+\frac{k D_{g f}}{\mu_{2}}\right)(1-c p)+c D_{c f l}\left(\frac{4 k p_{1}{ }^{2}}{\mu_{1}{ }^{2}}-\frac{\phi k D_{c f}{ }^{2} \dot{p}_{e}}{\mu_{2}}\right)}{\left(\phi D_{c j l}\left(\frac{2 D_{i f f} p_{1}}{\mu_{1}}+\frac{k D_{c f l} p_{c}}{\mu_{2}}\right)\right.} .
$$

We have not found a general solution for these equations but particular solutions can be obtained by numerical integration. For constant $p_{e}$, the solution is found easily. In this case, it can be shown that the only solution satisfying the initial conditions is the one for $p_{1}$ constant. Then Fq. D-15 reduces to

$$
\left(1-c p_{1}\right) \frac{p_{e}-p_{1}}{p_{1}}-\frac{2}{3} c p_{1} \frac{\mu_{2}}{\mu_{1}}=0 .
$$

For convenience, we let

$$
\xi=\frac{p_{p}-p_{1}}{p_{1}}
$$

Integration of Eq. D-10 then gives

$$
D_{i f f}=\sqrt{\frac{2 k p_{e}}{\phi \mu_{2}}\left[\frac{\xi\left(t-t_{o}\right)}{1+\xi}\right]} .
$$

Using this result in Eq. 15 gives Eq. 19. We find $\xi$ by substituting Eq. D-18 into Eq. D-17 to get

$$
\xi^{2}+\left(1-c p_{e}\right) \xi=\frac{2 c p_{e} \mu_{2}}{3 \mu_{1}},
$$

from which

$$
\xi=\frac{1}{2}\left[c p_{e}-1+\sqrt{\left(c p_{e}-1\right)^{2}+\frac{8 c p_{e} \mu_{2}}{3 \mu_{1}}}\right] .
$$

It is important to consider two special cases: when filtration enters empty pores and when the fracture fluid and reservoir fluid are the same, thus eliminating the moving boundary. In the first case, it is easily shown that, for $p_{e}$ constant, Eq. 15 and 16 give

$$
\dot{W}=\sqrt{\frac{k \phi p_{e}}{2 \mu\left(t-t_{o}\right)}}=\frac{C_{1}}{\sqrt{t-t_{o}}} .
$$

In the second case, the well-known result is

$$
\dot{W}=p_{e} \sqrt{\frac{k \phi c}{\pi \mu\left(t-t_{o}\right)}}=\frac{C_{2}}{\sqrt{t-t_{o}}} .
$$

These results have been used in the literature for many years to compute a leakoff coefficient $C_{t}$ from the relation

$$
\frac{1}{C_{t}}=\frac{1}{C_{1}}+\frac{1}{C_{2}} \text {. }
$$

Williams ${ }^{28}$ has derived a different relation,

$$
\frac{1}{C_{t}{ }^{2}}=\frac{1}{C_{1}{ }^{2}}+\frac{1}{C_{2} C_{t}} \text {, }
$$

by equating the sum of the pressure drops across $D_{i f l}$ and $D_{c f l}$ (Fig. 2) to the total pressure drop for fluid loss.

Leakoff velocities computed from our Eq. 20 are much different from those computed from either Eq. D-24 or D-25 in general. Eqs. D-24 and D-25 are derived by using Eqs. D-22 and D-23 to describe the viscous flow and compressibility mechanisms. This ignores the interdependence between the two phenomena that causes the boundary $D_{i f t}(t)$ to move. The moving boundary makes the leakoff problem a nonlinear one. This nonlinearity is expressed in Eqs. D-14, D-15, and D-16 but not in Eq. D-24 or D-25.

Next we consider the case of wall-building fluids with $W>W_{0}$. Combining Eqs. 15, D-10, and D-18 with Eq. 21 and letting

$$
\Lambda=\frac{\phi k}{\mu_{2}}\left(\frac{\xi}{1+\xi}\right) \text {, }
$$


we obtain

$$
p_{e}=\frac{p_{w}+\frac{W_{0}}{\iota} \dot{W}}{1+\Lambda} .
$$

Substituting this result in Eq. 20, we obtain

$$
\dot{W}^{2}=\frac{\Lambda}{1+\Lambda}\left[\frac{\left(p_{w^{\prime}}+W_{0} \dot{W}\right.}{2\left(t-t_{o}\right)}\right]
$$

which gives finally Eq. 22 .

\section{Appendix E}

We wish to derive Eq. 27 from Eq. 26. To begin, we note that the gradient $\partial p / \partial x$ can be treated as a body force acting on the fluid in the fracture. Under this body force, the virtual work associated with a variation $\delta u$ of the $x$ component of the fluid displacement is

$$
\delta W_{p}=\iiint-\frac{\partial p}{\partial x} \delta u \mathrm{~d} V=\int_{0}^{L_{f}}-\frac{\partial p}{\partial x} \delta V \mathrm{~d} x .
$$

The virtual displacement $\delta u$ must, of course, be compatible with the constraints. The variation $\delta V$ is given by

$$
\delta V=\frac{\partial V}{\partial L_{f}} \delta L_{f}+\frac{\partial V}{\partial b_{f}} \delta b_{f} .
$$

Substituting Eq. E-2 into Eq. E-1 gives

$$
\begin{aligned}
\delta W_{p} & =\left(\int_{0}^{L_{f}}-\frac{\partial p}{\partial x} \frac{\partial V}{\partial L_{f}} \mathrm{~d} x\right) \delta L_{f} \\
& -\left(\int_{0}^{L_{f}} \frac{\partial p}{\partial x} \frac{\partial V}{\partial b_{f}} \mathrm{~d} x\right) \delta b_{f} .
\end{aligned}
$$

Recognizing that fluid flow is the only source of dissipation, we have (from Eq. 23)

$$
\delta W_{p}=\mathrm{F}_{1} \delta L_{f}+\mathrm{F}_{2} \delta b_{f} .
$$

Comparing Eqs. E-4 and E-3, we derive

$$
\mathrm{F}_{1}=\int_{0}^{L_{f}}-\frac{\partial p}{\partial x} \frac{\partial V}{\partial b_{f}} \mathrm{~d} x=-L_{f} \int_{0}^{1} \frac{\partial p}{\partial x} \frac{\partial V}{\partial b_{f}} \mathrm{~d} L_{D}
$$

and

$$
\mathrm{F}_{2}=\int_{0}^{L_{f}}-\frac{\partial p}{\partial x} \frac{\partial V}{\partial b_{f}} \mathrm{~d} x=-L_{f} \int_{0}^{1} \frac{\partial p}{\partial x} \frac{\partial V}{\partial b_{f}} \mathrm{~d} L_{D}
$$

From Eq. A-12,

$$
\begin{aligned}
\mathrm{d} V & =2\left(L_{f} \mathrm{~d} b_{f}+b_{f} \mathrm{~d} L_{f}\right) \mathrm{F}\left(L_{D}\right) \\
& +2 b_{f} L_{D} \mathrm{f}\left(L_{D}\right) \mathrm{d} L_{f}, \ldots
\end{aligned}
$$

which gives

$$
\frac{\partial V}{\partial L_{f}}=2 b_{f}\left[\mathrm{~F}\left(L_{D}\right)-L_{D} \mathrm{f}\left(L_{D}\right)\right]
$$

$$
\frac{\partial V}{\partial b_{f}}=2 L_{f} \mathrm{~F}\left(L_{D}\right)
$$

or

$$
b_{f} \frac{\partial V}{\partial b_{f}}-L_{f} \frac{\partial V}{\partial L_{f}}=-2 L_{f} b_{f} L_{D} \mathrm{f}\left(L_{D}\right)
$$

Combining Eq. E-10 with Eqs. E-5 and E-6, we derive

$$
\mathrm{F}_{2} b_{f}-\mathrm{F}_{1} L_{f}=2 L_{f}{ }^{2} b_{f} \int_{0}^{l} \frac{\partial p}{\partial x} L_{D} \mathrm{f}\left(L_{D}\right) \mathrm{d} L_{D}
$$

Substituting Eq. E-11 into Eq. 26,

$$
E-2 L_{f} b_{f} \int_{0}^{1} \frac{\partial p}{\partial x} L_{D} \mathrm{f}\left(L_{D}\right)=2 K \gamma \frac{b_{f}^{2}}{L_{f}} .
$$

From Fq. A-19 we can write $b_{f}$ in terms of the total crack volume $V_{0}$.

$$
b_{f}=\frac{V_{0}}{2 \beta L_{f}} .
$$

Substituting this result into Eq. E-12 gives Eq. 27.

\section{SI Metric Conversion Factors}

$$
\begin{array}{ll}
\mathrm{ft} \times 3.048^{*} & \mathrm{E}-01=\mathrm{m} \\
\text { in. } \times 2.54^{*} & \mathrm{E}+00=\mathrm{cm} \\
\text { psi } \times 6.894757 & \mathrm{E}+00=\mathrm{kPa}
\end{array}
$$

"Conversion factor is exact.

SPEPE

Original manuscript received in the Society of Petroleum Engineers office Aug. 27 1982. Paper accepted for publication Aug. 10, 1984. Reviscd manuscript recoived Feb 14, 1985. Paper (SPE 11067) first presented at the 1982 SPE Annual Technical Conference and Exhibition held in New Orleans Sept. 26-29. 